\title{
Pereira desde los imaginarios de salud y actividad física de los usuarios de la Vía Activa, 2019
}

\author{
Stephany Aladino'; Sebastián Gutiérrez Quintín'; Nataly Vélez López'; \\ Stefanny Pérez Carmona'; Jhon Erick Martínez Palomino'; Natalia Marín Londoño'; \\ Claudia Jimena López García²; Jhonatan González Santamaría ${ }^{3}$
}

\begin{tabular}{l|l} 
RESUMEN & El siglo XXI conocido como el siglo del conocimiento, ha traído consigo una
\end{tabular} serie de cambios significativos en los modos y estilos de vida del grueso de los ciudadanos a lo largo del mundo. Nunca antes en la historia de la humanidad había existido tal nivel de acceso a información y comunicación como la hay hoy en día. El desarrollo tecnológico superó todas las expectativas posibles, y gracias a una economía de mercado a escala global, se hizo posible el intercambio no solo de productos y servicios, sino también de costumbres y hábitos, los cuales al menos en la salud pública han derivado un gran desafío en términos de cuidar la salud y preservar la vida de cara al significativo avance de las enfermedades no transmisibles (ENT).

Actualmente, la mitad de la población mundial se concentra en las ciudades a pesar de que estas solo ocupan el 3\% de la superficie habitable del planeta; por lo cual se hace necesario encaminar acciones que permitan identificar esas construcciones que realizan los ciudadanos a partir de sus vivencias y convivencias, entendiéndolas más allá de un espacio físico, como multiplicidad de imaginarios que toman sentido para individuos y colectivos.

En este orden de ideas, desde el programa de Fisioterapia de la Fundación Universitaria del Área Andina, se pretende desarrollar una investigación cualitativa de tipo hermenéutico, que permita estudiar los imaginarios de actividad física y salud en los usuarios de la Vía Activa de Pereira, como un mecanismo para generar información útil para los diferentes actores de la sociedad de cara al cumplimiento de las metas detalladas dentro de los objetivos de desarrollo sostenible: salud y bienestar, ciudades y comunidades sostenibles.

\begin{tabular}{l|l} 
PALABRAS & Actividad Física; \\
CLAVE & Ciclovía; \\
& Imaginario
\end{tabular}

1 Estudiante programa de Fisioterapia Fundación Universitaria del Área Andina.

2 Magister en Neurorehabilitación. Docente programa de Fisioterapia, Fundación Universitaria del Área Andina, clopez71@areandina.edu.co

3 Doctor en Ciencias de la Educación. Docente programa de Fisioterapia, Fundación Universitaria del Área Andina, jsantamaria13@areandina.edu.co 


\section{Pereira from the imaginary of health and physical activity of the users of the active route, 2019}

\begin{tabular}{l|l} 
ABSTRACT & $\begin{array}{l}\text { The } 21 \text { st century known as the century of knowledge has brought a series } \\
\text { of significant changes in the ways and lifestyles of the bulk of citizens } \\
\text { tnrougnout the world, never betore in the nistory of mankind nad such }\end{array}$ \\
a level of access to information and communication with what there is \\
today. Technological development exceeded all possible expectations and \\
thanks to a global market economy, made possible the exchange not only \\
of products and services, but also of customs and habits, which at least in \\
public health have derived a great challenge in terms of taking care of health \\
and preserving life for the significant advance of noncommunicable diseases \\
(NCDs).
\end{tabular}

Since currently half of the world's population is concentrated in cities, although they only occupy $3 \%$ of the habitable surface of the planet, it is necessary to direct actions that identify those constructions that citizens carry out from their lives and coexistence in the cities, thus understanding the cities beyond being a physical space, are a multiplicity of imaginary that make sense for individuals and groups.

In this order of ideas, from the Physiotherapy program of the University Foundation of the Andean Area it is intended to develop a qualitative research of a hermeneutical type that allows to study the imaginary of physical activity and health in the users of the Vía Activa de Pereira, as a mechanism to generate useful information for the different actors in society in order to meet the detailed goals within the objectives of sustainable development: health and well-being, and sustainable cities and communities.

Health;

Physical Activity;

KEYWORDS Bicycle Path; Imaginary 


\section{INTRODUCCIÓN}

Los imaginarios urbanos hacen referencia a las percepciones, creencias, diferentes estilos de vida y experiencias, que pueda tener una persona o un colectivo sobre alguna actividad, lugar o un suceso, frente al que cada uno de ellos está en condiciones de dar su opinión y ser replicada en diferentes personas, dando como resultado una percepción general de la población de cara a los diferentes acontecimientos, y así acercar y poner un punto de partida para las personas que deseen intervenir frente a alguna de estas situaciones. El estudio de los imaginarios urbanos abre una ventana para hacer aproximaciones comprensivas a esas construcciones colectivas que no son fácilmente cuantificables, pero que determinan las costumbres y formas de actuar en un contexto particular.

Las sociedades modernas presentan una característica poco favorable en términos de presencia de enfermedades. Las denominadas enfermedades no transmisibles (ENT) se han vuelto una constante a lo largo del planeta, y Colombia no es la excepción. Pese a las condiciones de violencia del país, el número de muertes por ENT es cada vez mayor y así mismo los costos asociados al sistema de salud.

En este orden se ideas, se hace necesario realizar una aproximación a los imaginarios urbanos de salud y actividad física, para comprender como facilitan, previenen o retrasan el desarrollo de ENT, y esta idea toma aun mayor relevancia cuando se tiene en cuenta que la Alcaldía de Pereira dispone de recursos y esfuerzos para el desarrollo de programas para la promoción de la salud y la actividad física, como es el caso de la Vía Activa.

A continuación, se presenta el desarrollo de un proceso investigativo cualitativo hermenéutico, el cual haciendo uso de una entrevista semiestructurada, permitirá la recolección de datos relacionada con las marcas urbanas, rutinas ciudadanas, temporalidades que configuran los imaginarios urbanos de salud y actividad física de los usuarios Vía Activa de Pereira.

\section{METODOLOGÍA}

Investigación cualitativa de tipo hermenéutico. En la actualidad la hermenéutica puede ser entendida como el arte del entendimiento a partir del diálogo. Dilthey afirmó que imaginar es interpretar comprensivamente, y comprender será el mecanismo para percibir la intención ajena(15). Se desarrollará una entrevista semiestructurada enfocada en dos componentes: salud y actividad física a población mayor de 15 años de edad asistente a la Vía Activa de la ciudad de Pereira. La muestra será de máxima variación, teniendo en cuenta como criterios de inclusión, el ser mayor de 15 años y usuario regular de Vía Activa. Los criterios de exclusión definidos son: presentar algún tipo de discapacidad que le impida responder preguntas de manera coherente y no residir en la ciudad de Pereira o Dosquebradas.

Población: población mayor de 15 años de edad asistente a la Vía Activa de la ciudad de Pereira 
Muestra: 379 personas.

Muestreo: máxima variación.

Descripción selección sujetos: los sujetos serán seleccionados aleatoriamente entre los usuarios de la Vía Activa.

Criterio de inclusión: ser mayor de 15 años - ser usuario de la Vía Activa.

Criterio de exclusión: Presentar discapacidad cognitiva o auditiva - No tener a Pereira como lugar de residencia permanente.

Descripción del método: se aplicarán entrevistas semiestructuradas a todos los sujetos participantes de la Vía Activa de la ciudad de Pereira que cumplan los criterios de inclusión. Las entrevistas se desarrollarán en tres puntos específicos de la Vía Activa ubicados equidistantemente.

Desarrollo del método: se realizará una sesión de inmersión inicial al campo en donde se probarán los instrumentos (formato de entrevistas y entrevistador). Posteriormente se realizará un análisis de la de los datos recolectados, así como de la experiencia de los entrevistadores para realizar posibles ajustes.

A continuación, se procederá a efectuar nuevamente entrevistas en la Vía Activa durante 3 domingos seguidos, en jornadas de 3 horas cada domingo. Con los datos recolectados durante los primeros tres domingos posteriores a la inmersión inicial, se realizará un proceso de codificación abierta para generar así las categorías que serán alimentadas en las sesiones posteriores a través de codificación axial. Una vez se saturen las categorías, se detendrá la recolección de datos y se procederá al análisis de datos final.

\section{RESULTADOS ESPERADOS}

En la actualidad el proyecto se encuentra en la fase de diseño de instrumentos, para lo cual el equipo de investigación está realizando adaptaciones de la metodología desarrollada por Silva, para garantizar la inclusión de preguntas que permitan realizar una aproximación comprensiva a las cualidades, calificaciones, marcas, rutinas y temporalidades relacionadas con los imaginarios urbanos de actividad física y salud.

\section{CONCLUSIÓN}

Los imaginarios urbanos se convierten en una categoría de investigación que se pone al servicio de la salud pública, pues a través de estos se puede hacer un acercamiento

\section{IMPACTOS}

El proyecto generará conocimiento valioso para las organizaciones sociales y las dependencias administrativas que participan activamente en la consolidación de espacios ciudadanos para la convivencia, la promoción de la salud y el desarrollo de ciudad 
1. Silva A. La ciudad marcada: los territorios urbanos. Imaginarios Urbanos [Internet]. 2006;26-41. Available from: http://medcontent.metapress.com/ index/A65RM03P4874243N.pdf\%5 1 Cnhttp://scholar.google.com/scholar?hl=e n\&btnG=Search\&q=intitle:Imaginarios+Urbanos $\# 0 \% 5 \mathrm{Cnhttp://scholar.google.}$ $\underline{\text { com/scholar?hl=en\&btnG=Search\&q=intitle:Imaginarios+urbanos } \# 0}$

2. Cabra F, Marciales G, Gualteros N, Mancipe E. Dimensiones socioculturales de la competencia informacional enlestudiantes universitarios: creencias, cultura académica ylexperiencias vitales. Rev. Iberoam Educ. 2011;(56/4):1-10

3. GiménezG. Modernización eidentidades sociales. 1994;12.Available from:http:// ru.iis.sociales.unam.mx:8080/jspui/bitstream/IIS/5012/1Modernizacion_e identidades_sociales.pdf

4. OXFAM. Una economía al servicio del 1\%. 2016

5. Organización Mundial de la Salud. Informe sobre la situación mundial de las enfermedades no transmisibles [Internet]. Who. 2014. Available from: http:// www.javeriana.edu.co/documents/12789/4434885/Carga+de+Enfermedad+Col ombia+2010.pdf/e0dbfe7b-40a2-49cb-848e-bd67bf7bc62e

6. Salud OP de la. La carga de las enfermedades en las Américas. Vol. 871. 2015

7. Ramírez campos Fernando, Rodríguez Andrea Martínez Mancel. Inequidades en Salud de las Enfermedades no Transmisibles en Colombia. 2013;1-60. Available from: https://www.minsalud.gov.co/sites/rid/Lists/BibliotecaDigital/RIDE/VS/ PP/ENT/inequidades-salud-enfermedades-no-transmisibles-colombia.pdf

8. Comisión Económica para América Latina y el Caribe - CEPAL. Panorama Social de América Latina, 2017. Organización de las Naciones Unidas -ONU. 2018. 1-203

9. Ministerio de Salud y de la Protección Social. Orientaciones Para el desarrollo de Ciudades, Entornos y Ruralidad Saludable (CERS). Orientaciones para el Desarrollo Ciudad Entornos y Rural Saludable. 2017;35.

10. Velásquez Bonilla N. Imaginarios de ciudad saludable. 2011;92. Available from: http://repositorio.utp.edu.co/dspace/handle/11059/2935

11. Llorca E, Amor MT, Merino B, Márquez FJ, Gómez F, Ramírez R. Ciudades saludables: Una estrategia de referencia en las políticas locales de salud pública. Gac Sanit. 2010;24(6):435-6.

12. Naciones Unidas/CEPAL. Agenda 2030 y los Objetivos de Desarrollo Sostenible: una oportunidad para América Latina y el Caribe. Publicación las Nac Unidas [Internet]. 2016; Mayo:50. Available from: http://www.sela.org/ media/2262361/agenda-2030-y-los-objetivos-de-desarrollo-sostenible.pdf

13. Ardila L, Rico J, Vélez A, Zuluaga J. Pereira Imaginada [Internet]. Universidad Tecnológica de Pereira; 2009. Available from: http:// repositorio.utp.edu.co/dspace/bitstream/handle/11059/1518/986132A676. pdf? sequence $=1$ \&isAllowed $=\mathrm{y}$

14. Peirce CS, Bugni B. La ciencia de la semiótica. 1974;109. Available from: http://mastor.cl/blog/wp-content/uploads/2015/08/PEIRCE-CH.-S.-La-Cienciade-La-Semiótica.pdf

15. Restrepo M. Representación Interpretante, enlace hermenéutico. RepInter. $2011 ; 25$

16. Maturana H. La realidad ¿objetiva o construida? 1996. p. 288. 